\title{
A long non-coding RNA signature for predicting survival in patients with colorectal cancer
}

\author{
Yi-Lin Wang ${ }^{1, *}$, Jun Shao ${ }^{3, *}$, Xiaohong $\mathrm{Wu}^{4,}{ }^{4}$, Tong $\mathrm{Li}^{5}$, Ming $\mathrm{Xu}^{3}$ and Debing Shi ${ }^{2}$ \\ ${ }^{1}$ Department of Hepatic Surgery, Fudan University Shanghai Cancer Center, Department of Oncology, Shanghai Medical \\ College, Fudan University, Shanghai 200032, China \\ ${ }^{2}$ Department of Colorectal Surgery, Fudan University Shanghai Cancer Center, Department of Oncology, Shanghai Medical \\ College, Fudan University, Xuhui District, Shanghai 200032, China \\ ${ }^{3}$ Department of General Surgery, Tongren Hospital, Shanghai Jiao Tong University School of Medicine, Changning District, \\ Shanghai 200336, China \\ ${ }^{4}$ Department of General Surgery, The Affiliated Yixing Hospital of Jiangsu University, Yixing, Jiangsu 214200, China \\ ${ }^{5}$ Department of General Surgery, Jinan Fourth People's Hospital, Jinan 250031, China \\ *These authors contributed equally to this work \\ Correspondence to: Debing Shi, email: shidebing7819@163.com \\ Ming XU, email: xuming925aero@gmail.com
}

Keywords: long non-coding RNA; prognosis; colorectal cancer

Received: July 05, 2017 Accepted: November 17, 2017 Epub: December 19, 2017 Published: April 24, 2018

Copyright: Wang et al. This is an open-access article distributed under the terms of the Creative Commons Attribution License 3.0 (CC BY 3.0), which permits unrestricted use, distribution, and reproduction in any medium, provided the original author and source are credited.

\section{ABSTRACT}

Dysregulation of long non-coding RNA (IncRNA) plays important roles in cancer development and progression. In this work, we attempted to develop a IncRNA signature to improve prognosis prediction of colorectal cancer. A comprehensive analysis for the IncRNA expression and corresponding clinical information of 344 colorectal patients has been performed based on the data from The Cancer Genome Atlas (TCGA). We randomly divided TCGA data into a training set $(n=172)$ and a testing set $(n=172)$. A four-IncRNA signature has been established which was significantly associated with the overall survival of colorectal cancer patients. Based on the four-IncRNA signature, the training set can be classified into high-risk and low-risk groups with significantly different survival. The result can be further validated in the testing dataset and another independent dataset. Further analyses suggested that the prognostic power of the four-IncRNA signature was independent of other clinical variables. The identification of IncRNA signature indicated that IncRNAs could be novel independent biomarkers for predicting the survival in patients with colorectal cancer.

\section{INTRODUCTION}

Colorectal cancer (CRC) is the third most common malignancy, and is the major cause of cancer-related death worldwide $[1,2]$. The incidence of colorectal cancer is gradually increasing in the developed areas. To date, surgery followed by adjuvant therapy is still the most common option for CRC patients. Despite an improved understanding of the molecular mechanism of CRC, the overall survival (OS) of CRC patients has not been dramatically improved and the four-year survival rate remains very low [3]. It is an urgent need to identify novel independent biomarkers for the diagnostic and prognosis of CRC.
With the advancements of transcriptome profiling, the roles of long non-coding RNAs (lncRNAs) have received great attention in the development of human cancer researches. LncRNAs are an important category of non-coding RNAs with little or no protein-coding capacity $[4,5]$. It has been documented that lncRNAs play important roles in regulating gene expression at transcriptional, posttranscriptional and epigenetic levels [4, 6-8]. Moreover, lncRNAs can participate in various biological processes and pathways, such as cell growth and immune response $[7,9,10]$. Recently, many lncRNAs have been examined to play critical oncogenic or tumor suppressive roles in various types of cancers [11-14]. 
Furthermore, several IncRNAs have been identified to be novel independent biomarkers for cancer prognosis [15-20]. As for colorectal cancer, recent studies have also revealed that some lncRNAs, such as PANDR, AFAP1$A S 1$ and TUG1, are dysregulated in CRC patients and play important role in the tumorigenesis [21-25].

We here attempted to develop a lncRNA signature to improve prognosis prediction of CRC. We identified a fourlncRNA signature by using the sample-splitting method. Our results demonstrated the four-lncRNA signature can provide a novel insight into the understanding of the underlying molecular mechanism of CRC.

\section{RESULTS}

\section{Identification of prognostic IncRNAs from the training dataset}

The 344 CRC patients were randomly divided into a training dataset $(n=172)$ and a testing dataset $(n=172)$. At first, we identified the prognostic lncRNAs from the training set. A univariate Cox regression analysis was performed to evaluate the association between lncRNA expression and overall survival of CRC patients. Based on the threshold of $P$-value $<0.01$, four lncRNAs were identified to be significantly correlated with overall survival of CRC patients. The detailed information of these four lncRNAs was showed in Table 1. Positive coefficients represent that higher expression profiles were associated with shorter overall survival (SPRY4IT1), whereas negative coefficients represent that higher expression level of lncRNA expression was associated with longer survival (LINC01133, Loc554202 and RP11$727 F 15.13)$.

\section{A four-IncRNA signature for predicting overall survival of CRC patients}

These four lncRNAs were analyzed using a multivariate Cox regression analysis to establish a lncRNA signature for predicting patients' overall survival. We constructed a risk-score formula by integrating the lncRNA expressions and corresponding estimated regression coefficient derived from above multivariate Cox regression analysis, as follows: Risk score $=(0.322$ $\times$ expression value of SPRY4-IT1 $)+(-0.134 \times$ expression value of Loc554202) $+(-0.336 \times$ expression value of LINC01133 $)+(-0.231 \times$ expression value of RP11$727 F 15.13)$. We calculated four-lncRNA signature risk score for each CRC patient, and ranked them according to risk score values. These $172 \mathrm{CRC}$ patients can be divided into a high-risk group $(n=90)$ and a low-risk group $(n=82)$ using the median risk score as the threshold.

A significant difference of overall survival between the high-risk group and low-risk group was observed $(P$-value $=1.74 \mathrm{E}-06$; Figure $1 \mathrm{~A}) . \mathrm{It}$ is obvious that $\mathrm{CRC}$ patients in the high-risk group had significantly shorter survival (median 18 months) than those in the lowrisk group (median 24.5 months). The time-dependent ROC curve analysis achieved an AUC of 0.727 at the overall survival of five years (Figure 1B), suggesting a competitive performance of the four-lncRNA signature for survival prediction. The lncRNA risk score were significantly associated with overall survival of CRC patients using the univariate Cox regression analysis (Table 2).

\section{Validation of the four-lncRNA signature for survival prediction in the testing dataset and another independent dataset}

We confirmed our results using the testing set. Using the same risk score formula, $172 \mathrm{CRC}$ patients can be classified into a high-risk group $(n=77)$ and a low-risk group ( $n=95)$ with the same cutoff point derived from the training dataset. The result showed that a significant difference of overall survival between the high-risk group and the low-risk group $(P$-value $=0.00439$, median 17.5 months vs. 23 months; Figure 2A). The AUC value in the testing set was 0.712 at the overall survival of four years, and the lncRNA risk score was significantly associated with patients' overall survival (Table 2). Next, we performed the same analysis in the entire TCGA CRC dataset. similar results were obtained. The lncRNA signature can classify 344 CRC patients into a high-risk group $(n=166)$ and a low-risk group $(n=178)$ with significant difference of overall survival $(P$-value $=6.9 \mathrm{E}-05$, median 16 months vs. 23 months; Figure 2B). The AUC value in the entire set was 0.721 at the overall survival of four years. Further analysis indicated that lncRNA risk score was significantly associated with CRC patients' overall survival in the entire TCGA CRC dataset (Table 2). We further validated our lncRNA signature in an independent CRC data (GSE14333). As shown in Figure 2C, lncRNA signature can effectively predict overall survival in CRC patients. A significant difference of overall survival between the high-risk group $(n=125)$ and the low-risk group $(n=72)$ was observed $(P$-value $=0.0183$, median 38.3 months $v s$. 58.3 months).

\section{Independence of the IncRNA signature for survival prediction from other clinical variables}

We examined whether the prognostic power of the lncRNA signature was independent of other clinical variables, such as age, gender, subtype and tumor stage. The multivariate Cox regression analyses were performed, and the results suggested that the lncRNA risk score was also significantly associated with overall survival. The lncRNA signature still maintained a significant association with overall survival after adjustment for other clinical variables (Table 2). The result showed that patient age 
Table 1: The detailed information of four prognostic IncRNAs significantly associated with overall survival in patients with CRC

\begin{tabular}{lccc}
\hline Gene symbol & $\boldsymbol{P}_{\text {value }}^{\mathbf{a}}$ & Hazard ratio $^{\mathbf{a}}$ & Coefficient $^{\mathbf{b}}$ \\
\hline SPRY4-IT1 & $3.71 \mathrm{E}-04$ & 1.637 & 0.322 \\
RP11-727F15.13 & $2.88 \mathrm{E}-03$ & 0.746 & -0.231 \\
Loc554202 & $6.38 \mathrm{E}-03$ & 0.560 & -0.134 \\
LINC01133 & $1.39 \mathrm{E}-04$ & 0.751 & -0.336 \\
\hline
\end{tabular}

${ }^{\mathrm{a}, \mathrm{b}}$ Derived from the univariate and multivariate Cox regression analyses in CRC patients of the training dataset.

and tumor stage were significantly associated with overall survival. A series stratified analyses have been performed according to age and tumor stage, respectively. At first, all CRC patients were stratified into a younger group $(n=132$, age $<65)$ and an elder group $(n=212$, age $\geq 65)$. The IncRNA signature can divided the younger group into a high-risk subgroup $(n=85)$ and a low-risk subgroup $(n=47)$ with significant difference of survival $(P$-value $=0.00416$, median 23 months vs. 50.85 months; Figure 3A). As for the elder group, the four-lncRNA signature was also able to classify them into a high-risk subgroup $(n=147)$ and a low-risk subgroup $(n=65)$ with significantly different survival $(P$-value $=0.00742$, median 13.3 months vs. 20.1 months; Figure 3B). Next, all CRC patients were stratified by tumor stage into an early subgroup (stage I and II, $n=196$ ) and a late subgroup (stage III and IV, $n=148$ ), respectively. The result of stratified analysis showed effective prognostic power in both early subgroup and late subgroup. As shown in Figure 4A, patients in the early subgroup can be divided into a high-risk group $(n=92)$ with shorter survival and a low-risk group $(n=104)$ with longer survival $(P$-value $=0.00189$, median 26 months vs. 51.05

A

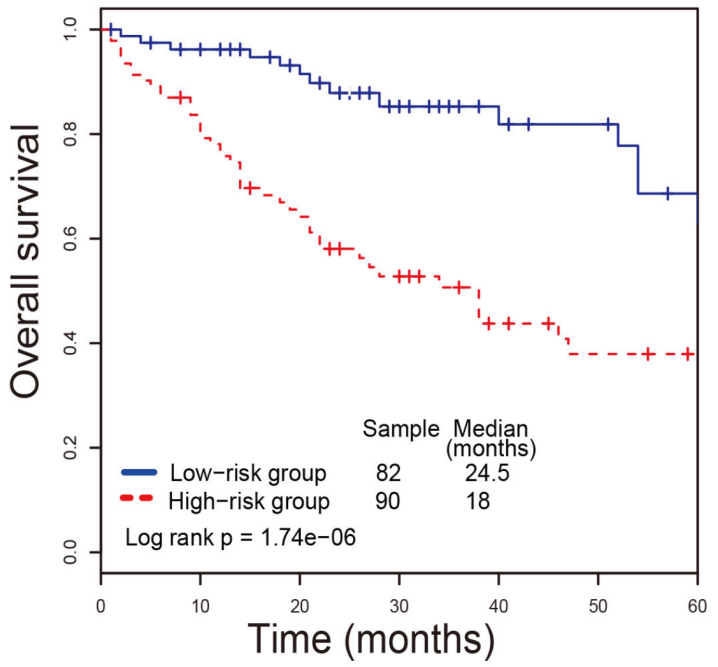

months). Similar results were obtained in the late subgroup $(P$-value $=2.48 \mathrm{E}-04$, median 16 months vs. 24.5 months; Figure 4B). These result demonstrated that the prognostic ability of IncRNA signature is independent of other clinical variables for the prediction of survival in CRC patients.

\section{Functional implications of the prognostic IncRNAs}

We investigated the potential functional roles of the four prognostic lncRNAs in CRC. Spearman correlation coefficients were calculated between IncRNAs and protein-coding genes using the expression profiles of 344 CRC patients. A total of 732 protein-coding genes were positively correlated with either of the four lncRNAs (Spearman correlation coefficient $>0.6$ ). Functional enrichment analyses indicated that these protein-coding genes were significantly enriched in $20 \mathrm{GO}$ categories ( $P$-value of $<0.01$, Figure 5 ). These functionally enriched GO categories included assembly and disassembly of protein and macromolecules, transcription, signal transduction and response to stimulus, cell apoptosis and

B

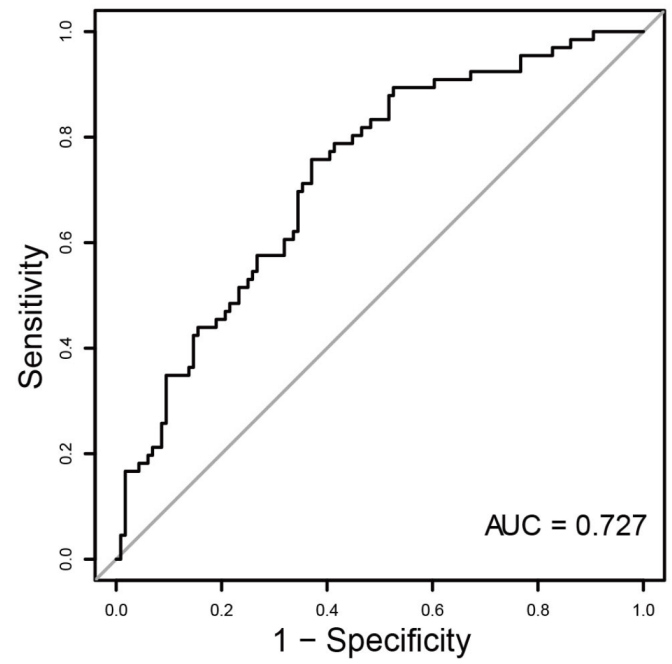

Figure 1: The four-IncRNA signature in prognosis of survival of CRC patients in the training dataset. (A) The KaplanMeier curves of overall survival between high-risk and low-risk patients in the training dataset. (B) The ROC curve for survival prediction by the four-lncRNA signature within four years as the defining point. 
Table 2: Univariate and multivariate cox regression analyses in each dataset

\begin{tabular}{|c|c|c|c|c|c|c|}
\hline \multirow{2}{*}{ Variables } & \multicolumn{3}{|c|}{ Univariate analysis } & \multicolumn{3}{|c|}{ Multivariate analysis } \\
\hline & HR & $95 \%$ CI of HR & $P$ value & HR & $95 \%$ CI of HR & $P$ value \\
\hline \multicolumn{7}{|c|}{ Training dataset $(n=172)$} \\
\hline \multicolumn{7}{|c|}{ Four-lncRNA risk score } \\
\hline Low risk/High risk & 17.25 & $1.28-29.40$ & $1.37 \mathrm{E}-05$ & 21.62 & $1.34-32.28$ & $6.83 \mathrm{E}-05$ \\
\hline \multicolumn{7}{|l|}{ Age } \\
\hline$\leq 65 />65$ & 1.74 & $0.54-4.91$ & 0.006 & 1.53 & $0.37-3.70$ & 0.0042 \\
\hline \multicolumn{7}{|l|}{ Gender } \\
\hline Female/Male & 0.826 & $0.38-4.61$ & 0.62 & 0.91 & $0.51-2.78$ & 0.73 \\
\hline \multicolumn{7}{|l|}{ Stage } \\
\hline II & 1 (reference) & & & 1 (reference) & & \\
\hline III/IV & 2.18 & $0.58-4.71$ & 0.012 & 2.24 & $0.36-4.95$ & 0.01 \\
\hline \multicolumn{7}{|c|}{ Testing dataset $(n=172)$} \\
\hline \multicolumn{7}{|c|}{ Four-lncRNA risk score } \\
\hline Low risk/High risk & 3.33 & $1.42-5.71$ & $3.01 \mathrm{E}-03$ & 2. 59 & $1.07-5.89$ & $6.48 \mathrm{E}-03$ \\
\hline \multicolumn{7}{|l|}{ Age } \\
\hline$\leq 65 />65$ & 1.20 & $0.48-5.74$ & 0.006 & 183 & $0.45-3.24$ & 0.008 \\
\hline \multicolumn{7}{|l|}{ Gender } \\
\hline Female/Male & 1.49 & $0.43-2.95$ & 0.67 & 1.23 & $0.44-3.16$ & 0.62 \\
\hline \multicolumn{7}{|l|}{ Stage } \\
\hline II & 1 (reference) & & & 1 (reference) & & \\
\hline III/IV & 1.65 & $0.55-5.44$ & 0.021 & 1.49 & $0.42-5.11$ & 0.033 \\
\hline \multicolumn{7}{|c|}{ Entire dataset $(n=344)$} \\
\hline \multicolumn{7}{|c|}{ Four-lncRNA risk score } \\
\hline Low risk/High risk & 5.56 & $2.81-11.72$ & 6. $8 \mathrm{E}-04$ & 4.98 & $2.54-9.88$ & $8.42 \mathrm{E}-04$ \\
\hline \multicolumn{7}{|l|}{ Age } \\
\hline$\leq 65 />65$ & 1.12 & $0.603-4.03$ & 0.01 & 1.29 & $0.63-5.48$ & 0.01 \\
\hline \multicolumn{7}{|l|}{ Gender } \\
\hline Female/Male & 0.74 & $0.51-1.95$ & 0.35 & 1.45 & $0.56-2.9$ & 0.54 \\
\hline \multicolumn{7}{|l|}{ Stage } \\
\hline II & 1 (reference) & & & 1 (reference) & & \\
\hline III/IV & 2.92 & $1.26-5.85$ & 0.01 & 1.66 & $0.74-5.42$ & 0.01 \\
\hline
\end{tabular}

death, metabolic and catabolic process, cell cycle, DNA replication and DNA repair, etc. The result suggested that the four prognostic lncRNAs may participate in CRC tumorigenesis through regulating protein-coding genes to influence CRC-related biological pathways.

\section{DISCUSSION}

Great efforts have been devoted to detect prognostic biomarkers for $\mathrm{CRC}$ at protein-coding and non-coding genes [21, 22, 26, 27]. Mounting evidence suggested that expression changes of lncRNAs are implicated in tumorigenesis by acting as tumor oncogenes or suppressor [8, 28]. Moreover, dysregulation of lncRNA has been measured in various cancer types, highlighting their potential roles as novel independent biomarkers for cancer prognosis [10, 29-32]. Some works have identified potential prognostic lncRNA signatures to predict overall survival in many cancer types, such as glioblastoma, lung cancer, etc. $[15,18]$. However, the prognostic power of lncRNA signature for predicting survival in patients with CRC has still not been investigated.

Up to date, many lncRNAs have been discovered in human over the past decades [33]. However, only few of them are well characterized in human cancers. Among these four lncRNAs, SPRY4-IT1 and LINC01133 
have been reported to be prognostic factors in patients with CRC $[34,35]$. In this work, we identified that four lncRNAs are significantly associated with CRC patients' survival and established a four-lncRNA signature for the prediction of survival. The result suggested a competitive performance of four-lncRNA signature for predicting survival. This finding can be validated by using TCGA testing set and another independent dataset, which demonstrated the reliability and reproducibility of the four-lncRNA signature for predicting CRC patients' survival. Further stratified analyses after controlling for age and tumor stage showed that the prognostic power of the four-lncRNA signature was independent of other clinical variables for survival prediction of patients with CRC.
Previous studies documented that IncRNAs participated in biological processes by positively regulating protein-coding genes involved in the same processes. It is possible to predict lncRNA biological functions based on their co-expressed protein-coding genes [36-38]. Here, we performed GO enrichment analyses for lncRNA co-expressed protein-coding genes. The results demonstrated the important functional roles of the four prognostic lncRNAs in CRC tumorigenesis.

Taken together, we performed a comprehensive analysis for lncRNA expression profiles and corresponding clinical information in CRC patients. Our work identified that four prognostic lncRNAs were significantly associated with CRC patients' survival. A four-lncRNA signature was established to effectively predict patients' survival.
A

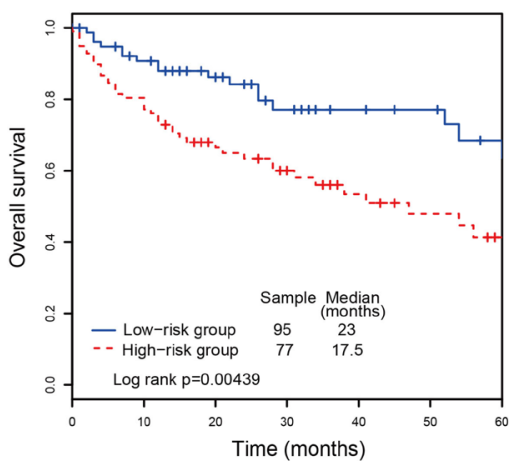

B

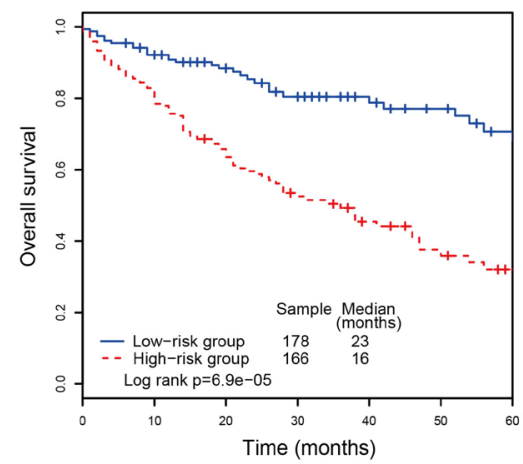

C

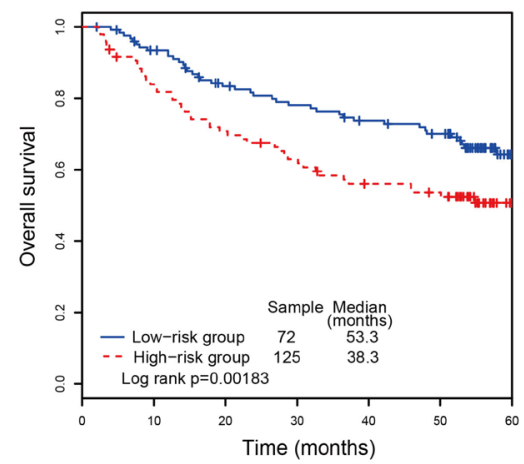

Figure 2: The Kaplan-Meier curves of overall survival between high-risk and low-risk patients in the testing, entire dataset and another independent dataset. (A) The Kaplan-Meier curves for the testing dataset. (B) The Kaplan-Meier curves for the entire dataset. (C) The Kaplan-Meier curves for the dataset from Gene Expression Omnibus database.

A

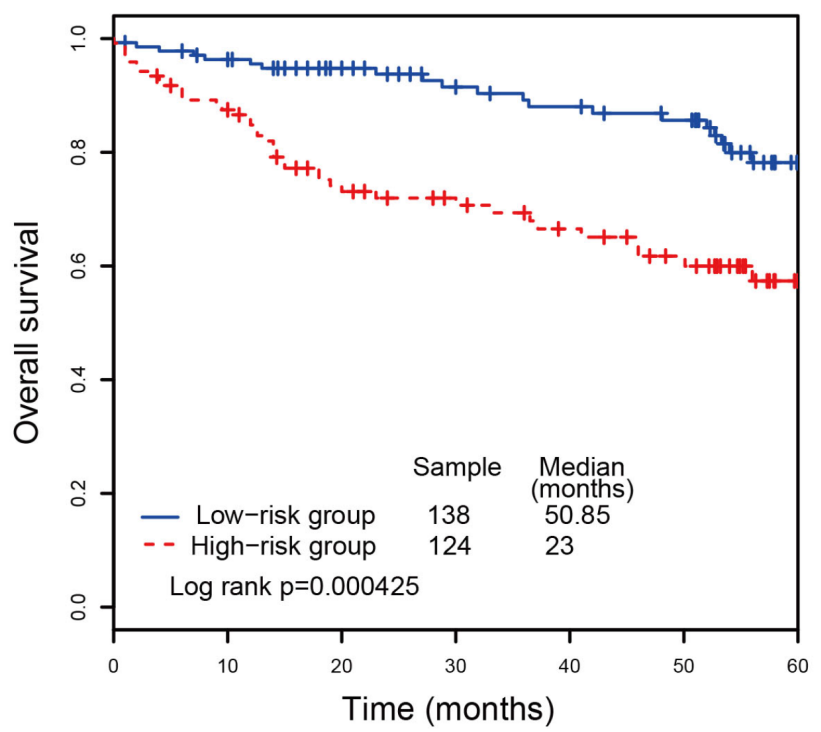

B

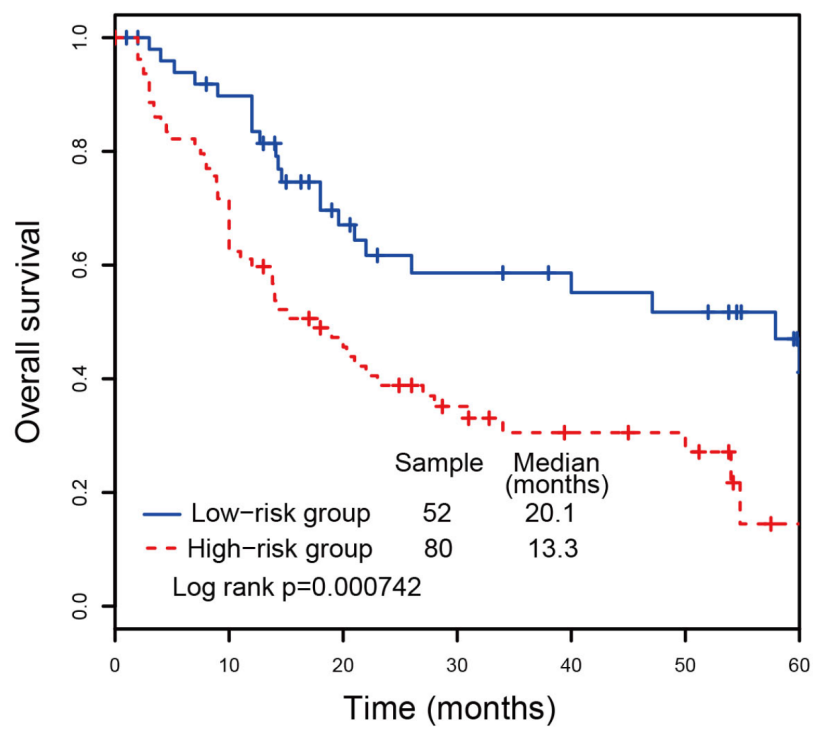

Figure 3: Survival analyses of all CRC patients stratified by age and tumor stage with the four-IncRNA signature. (A) The Kaplan-Meier curves for the younger dataset. (B) The Kaplan-Meier curves for the elder dataset. 
A

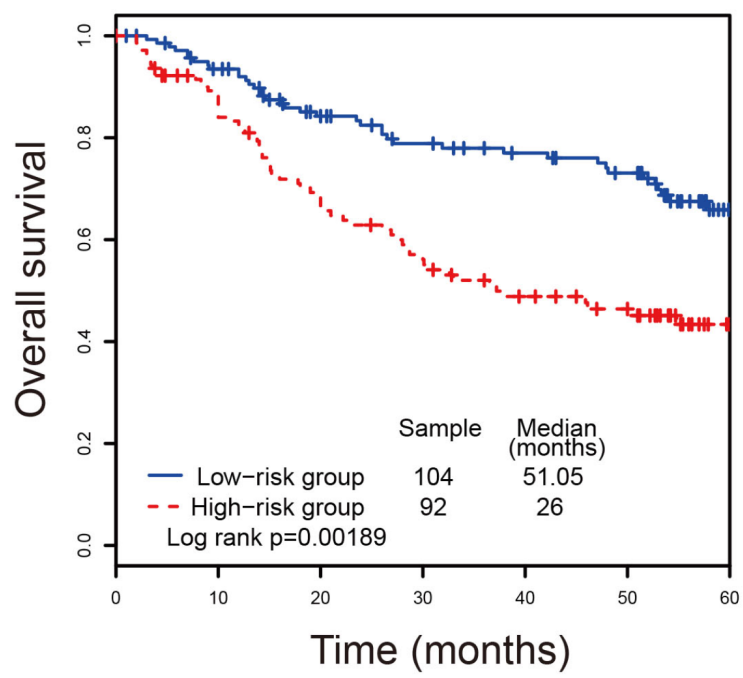

B

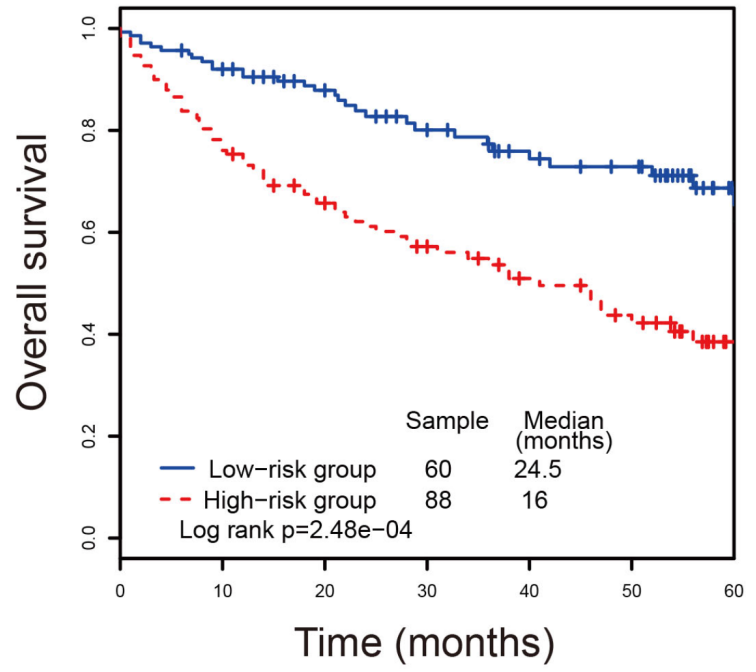

Figure 4: Survival analyses of all CRC patients stratified by tumor stage with the four-IncRNA signature. (A) The Kaplan-Meier curves for the early stage dataset. (B) The Kaplan-Meier curves for the late stage dataset.

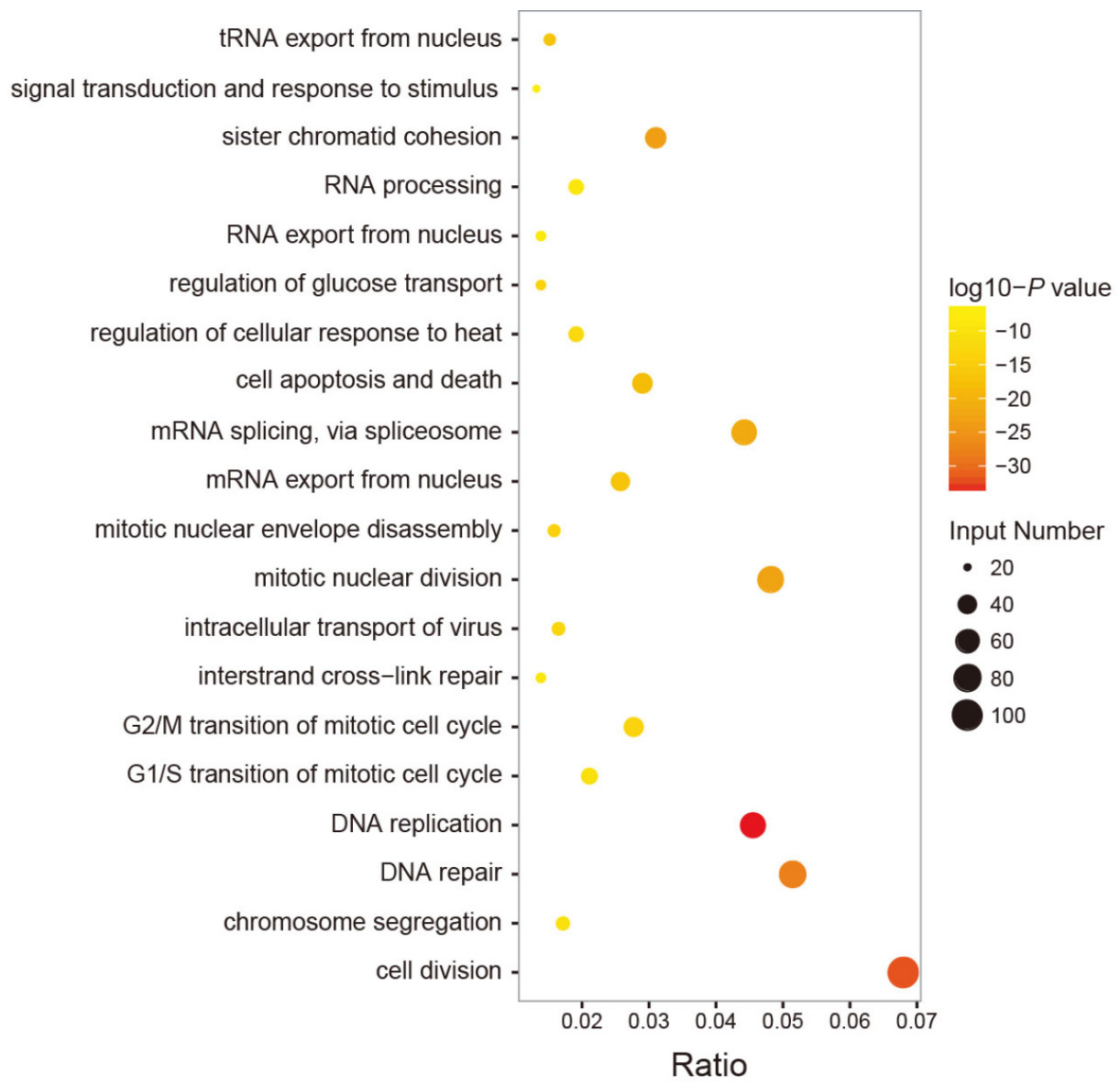

Figure 5: Functional enrichment analyses of the protein-coding genes co-expressed with the four prognostic IncRNAs. (A) The functional enrichment map of GO terms. Each node represents a GO category. An edge represents the overlap of the shared genes between connecting terms. Node size represents the number of gene in the GO terms. Color intensity is proportional to enrichment significance. 
The four-lncRNA signature might function as novel independent biomarkers for CRC prognosis. Our work gains insight into the understanding of the molecular mechanism of CRC.

\section{MATERIALS AND METHODS}

\section{CRC datasets and clinical information}

CRC IncRNA data and corresponding clinical information were downloaded from TCGA data portal. A total of 344 CRC patients were included in this work after removal of patients without clear clinical information. The lncRNAs derived from TCGA were annotated based on GENCODE database [39] to reduce redundant. The lncRNA expressions were defined as those with an average Fragments Per Kilobase of transcript per Million fragments mapped $(\mathrm{FPKM}) \geq 0.1$. The lncRNAs expression profiles were normalized by $\log 2$ transformed. At last, a total of 14,467 lncRNAs were enrolled in 344 CRC patients.

\section{Identification of prognostic IncRNA signature}

We randomly divided CRC patients into a training set $(n=172)$ and a testing set $(n=172)$. In this training set, the association between the lncRNA expression and the overall survival of CRC patients was evaluated using a univariate Cox regression analysis. The lncRNAs that are significantly associated with the overall survival of CRC patients were identified based on the threshold of $P$-value $<0.01$. Next, those selected lncRNAs were subjected to a multivariate Cox regression analysis. We established a risk score formula according to the IncRNA expression, weighted by the regression coefficients derived from the multivariable Cox regression analysis. Then, $\mathrm{CRC}$ patients in the training set can be divided into high-risk or low-risk groups by using the median risk score as a threshold.

The survival differences between high-risk and lowrisk group in each dataset can be evaluated by the KaplanMeier analyses. Multivariate Cox regression and stratified analyses were carried out to evaluate whether the prognostic power of the four-lncRNA signature was independent of other clinical variables. The receiver operating characteristic (ROC) curve analyses were performed to evaluate the competitive performance for overall survival prediction. Area under the ROC curve (AUC) values were calculated. All analyses were performed using $\mathrm{R}$ package.

\section{Functional enrichment analyses}

Since lncRNAs are always co-expressed with neighboring coding genes, we calculated spearman correlation coefficients to evaluate co-expression relationships between lncRNAs and protein-coding genes. Functional enrichment analyses for those co-expressed protein-coding genes were performed using the DAVID software [40, 41]. Gene Ontology (GO) categories with a $P$-value of $<0.01$ were considered as significantly enriched function annotations.

\section{Author contributions}

MX, YW and DS conceived and designed the experiments. YW, JS, XW, TL analyzed the data and wrote the manuscript. All authors read and approved the final manuscript.

\section{ACKNOWLEDGMENTS}

We thank all the subject of this work for their participation.

\section{CONFLICTS OF INTEREST}

The authors declare that they have no competing interests.

\section{REFERENCES}

1. Siegel RL, Miller KD, Jemal A. Cancer statistics, 2016. CA Cancer J Clin. 2016; 66:7-30.

2. Jemal A, Center MM, DeSantis C, Ward EM. Global patterns of cancer incidence and mortality rates and trends. Cancer Epidemiol Biomarkers Prev. 2010; 19:1893-1907.

3. Markle B, May EJ, Majumdar AP. Do nutraceutics play a role in the prevention and treatment of colorectal cancer? Cancer Metastasis Rev. 2010; 29:395-404.

4. Mercer TR, Dinger ME, Mattick JS. Long non-coding RNAs: insights into functions. Nat Rev Genet. 2009; 10:155-159.

5. Ponting CP, Oliver PL, Reik W. Evolution and functions of long noncoding RNAs. Cell. 2009; 136:629-641.

6. Kornienko AE, Guenzl PM, Barlow DP, Pauler FM. Gene regulation by the act of long non-coding RNA transcription. BMC Biol. 2013; 11:59.

7. Fatica A, Bozzoni I. Long non-coding RNAs: new players in cell differentiation and development. Nat Rev Genet. 2014; 15:7-21.

8. Gibb EA, Vucic EA, Enfield KS, Stewart GL, Lonergan KM, Kennett JY, Becker-Santos DD, MacAulay CE, Lam S, Brown CJ, Lam WL. Human cancer long non-coding RNA transcriptomes. PLoS One. 2011; 6:e25915.

9. Geisler S, Coller J. RNA in unexpected places: long noncoding RNA functions in diverse cellular contexts. Nat Rev Mol Cell Biol. 2013; 14:699-712.

10. Gutschner T, Diederichs S. The hallmarks of cancer: a long non-coding RNA point of view. RNA Biol. 2012; 9:703-719.

11. Tsai MC, Spitale RC, Chang HY. Long intergenic noncoding RNAs: new links in cancer progression. Cancer Res. 2011; 71:3-7. 
12. Uchida S, Dimmeler S. Long noncoding RNAs in cardiovascular diseases. Circ Res. 2015; 116:737-750.

13. Kumar V, Westra HJ, Karjalainen J, Zhernakova DV, Esko T, Hrdlickova B, Almeida R, Zhernakova A, Reinmaa E, Vosa U, Hofker MH, Fehrmann RS, Fu J, et al. Human disease-associated genetic variation impacts large intergenic non-coding RNA expression. PLoS Genet. 2013; 9:e1003201.

14. Wang P, Ren Z, Sun P. Overexpression of the long non-coding RNA MEG3 impairs in vitro glioma cell proliferation. J Cell Biochem. 2012; 113:1868-1874.

15. Zhang XQ, Sun S, Lam KF, Kiang KM, Pu JK, Ho AS, Lui WM, Fung CF, Wong TS, Leung GK. A long noncoding RNA signature in glioblastoma multiforme predicts survival. Neurobiol Dis. 2013; 58:123-131.

16. Meng J, Li P, Zhang Q, Yang Z, Fu S. A four-long noncoding RNA signature in predicting breast cancer survival. J Exp Clin Cancer Res. 2014; 33:84.

17. Hu Y, Chen HY, Yu CY, Xu J, Wang JL, Qian J, Zhang X, Fang JY. A long non-coding RNA signature to improve prognosis prediction of colorectal cancer. Oncotarget. 2014; 5:2230-2242. https://doi.org/10.18632/oncotarget.1895.

18. Zhou M, Guo M, He D, Wang X, Cui Y, Yang H, Hao D, Sun J. A potential signature of eight long non-coding RNAs predicts survival in patients with non-small cell lung cancer. J Transl Med. 2015; 13:231.

19. Zhou M, Zhao H, Wang Z, Cheng L, Yang L, Shi H, Yang H, Sun J. Identification and validation of potential prognostic lncRNA biomarkers for predicting survival in patients with multiple myeloma. J Exp Clin Cancer Res. 2015; 34:102.

20. Zhou M, Sun Y, Sun Y, Xu W, Zhang Z, Zhao H, Zhong Z, Sun J. Comprehensive analysis of lncRNA expression profiles reveals a novel lncRNA signature to discriminate nonequivalent outcomes in patients with ovarian cancer. Oncotarget. 2016; 7:32433-48. https://doi.org/10.18632/ oncotarget.8653.

21. Lu M, Liu Z, Li B, Wang G, Li D, Zhu Y. The high expression of long non-coding RNA PANDAR indicates a poor prognosis for colorectal cancer and promotes metastasis by EMT pathway. J Cancer Res Clin Oncol. 2017; 143:71-81.

22. Han X, Wang L, Ning Y, Li S, Wang Z. Long non-coding RNA AFAP1-AS1 facilitates tumor growth and promotes metastasis in colorectal cancer. Biol Res. 2016; 49:36.

23. Wang L, Zhao Z, Feng W, Ye Z, Dai W, Zhang C, Peng J, $\mathrm{Wu} \mathrm{K}$. Long non-coding RNA TUG1 promotes colorectal cancer metastasis via EMT pathway. Oncotarget. 2016; 7:51713-51719. https://doi.org/10.18632/oncotarget.10563.

24. Damas ND, Marcatti M, Come C, Christensen LL, Nielsen MM, Baumgartner R, Gylling HM, Maglieri G, Rundsten CF, Seemann SE, Rapin N, Thezenas S, Vang S, et al. SNHG5 promotes colorectal cancer cell survival by counteracting STAU1-mediated mRNA destabilization. Nat Commun. 2016; 7:13875.
25. Yang ZY, Yang F, Zhang YL, Liu B, Wang M, Hong X, Yu Y, Zhou YH, Zeng H. LncRNA-ANCR down-regulation suppresses invasion and migration of colorectal cancer cells by regulating EZH2 expression. Cancer Biomark. 2017; 18:95-104.

26. Ragusa M, Barbagallo C, Statello L, Condorelli AG, Battaglia R, Tamburello L, Barbagallo D, Di Pietro C, Purrello M. Non-coding landscapes of colorectal cancer. World J Gastroenterol. 2015; 21:11709-11739.

27. Ren YK, Xiao Y, Wan XB, Zhao YZ, Li J, Li Y, Han GS, Chen XB, Zou QY, Wang GC, Lu CM, Xu YC, Wang YC. Association of long non-coding RNA HOTTIP with progression and prognosis in colorectal cancer. Int J Clin Exp Pathol. 2015; 8:11458-11463.

28. Qiu MT, Hu JW, Yin R, Xu L. Long noncoding RNA: an emerging paradigm of cancer research. Tumour Biol. 2013; 34:613-620.

29. Zhang H, Chen Z, Wang X, Huang Z, He Z, Chen Y. Long non-coding RNA: a new player in cancer. J Hematol Oncol. $2013 ; 6: 37$.

30. Cabili MN, Trapnell C, Goff L, Koziol M, Tazon-Vega B, Regev A, Rinn JL. Integrative annotation of human large intergenic noncoding RNAs reveals global properties and specific subclasses. Genes Dev. 2011; 25:1915-1927.

31. Brunner AL, Beck AH, Edris B, Sweeney RT, Zhu SX, Li R, Montgomery K, Varma S, Gilks T, Guo X, Foley JW, Witten DM, Giacomini CP, et al. Transcriptional profiling of long non-coding RNAs and novel transcribed regions across a diverse panel of archived human cancers. Genome Biol. 2012; 13:R75.

32. Gibb EA, Brown CJ, Lam WL. The functional role of long non-coding RNA in human carcinomas. Mol Cancer. 2011; $10: 38$.

33. Hangauer MJ, Vaughn IW, McManus MT. Pervasive transcription of the human genome produces thousands of previously unidentified long intergenic noncoding RNAs. PLoS Genet. 2013; 9:e1003569.

34. Tan W, Song ZZ, Xu Q, Qu X, Li Z, Wang Y, Yu Q, Wang S. Up-Regulated Expression of SPRY4-IT1 Predicts Poor Prognosis in Colorectal Cancer. Med Sci Monit. 2017; 23:309-314.

35. Zhang JH, Li AY, Wei N. Downregulation of long noncoding RNA LINC01133 is predictive of poor prognosis in colorectal cancer patients. Eur Rev Med Pharmacol Sci. 2017; 21:2103-2107.

36. Liao Q, Liu C, Yuan X, Kang S, Miao R, Xiao H, Zhao G, Luo H, Bu D, Zhao H, Skogerbo G, Wu Z, Zhao Y. Largescale prediction of long non-coding RNA functions in a coding-non-coding gene co-expression network. Nucleic Acids Res. 2011; 39:3864-3878.

37. Guo X, Gao L, Liao Q, Xiao H, Ma X, Yang X, Luo H, Zhao G, Bu D, Jiao F, Shao Q, Chen R, Zhao Y. Long non-coding RNAs function annotation: a global prediction 
method based on bi-colored networks. Nucleic Acids Res. 2013; 41:e35.

38. Ma H, Hao Y, Dong X, Gong Q, Chen J, Zhang J, Tian W. Molecular mechanisms and function prediction of long noncoding RNA. ScientificWorldJournal. 2012; 2012:541786.

39. Harrow J, Frankish A, Gonzalez JM, Tapanari E, Diekhans M, Kokocinski F, Aken BL, Barrell D, Zadissa A, Searle S, Barnes I, Bignell A, Boychenko V, et al.
GENCODE: the reference human genome annotation for The ENCODE Project. Genome Res. 2012; 22:1760-1774.

40. Huang da W, Sherman BT, Lempicki RA. Systematic and integrative analysis of large gene lists using DAVID bioinformatics resources. Nat Protoc. 2009; 4:44-57.

41. Huang da W, Sherman BT, Lempicki RA. Bioinformatics enrichment tools: paths toward the comprehensive functional analysis of large gene lists. Nucleic Acids Res. 2009; 37:1-13. 